clusion of individual photographs of the participants, which does much to preserve the spirit of the occasion and its international flavour. This is an original idea and one which is worth adopting elsewhere.

Apart from the first four papers on general problems of psychopharmacology, which make an effective introduction to the subject, the remainder of the book is divided equally into three parts. One deals with specific problems, mainly of methodology, and the other two cover practical and theoretical issues respectively. The papers are of unequal merit but all are relevant. There is a full account of the discussions which followed the three sessions of the symposium, a technical appendix on the use of the analogue computor in studies of thyroid function, and a relatively long paper on the psychic action of the two major drugs which was not read at the meeting. There is no index, and references follow the individual papers.

The title of the book gives pretence of greater scope than the book actually possesses, and we look in vain for adequate treatment of the hallucinogens or 5-hydroxytryptamine, the two most important lines of research into neurochemistry and the aetiology of the psychoses. Taking the work for what it is, however-a study of reserpine and chlorpromazine in contemporary research and clinical application-we find it makes an important contribution to the literature on psychotropic medication.

Symposium publications are a new venture in medical bibliography: lacking the balanced gestalt of the textbook, their value lies in the technical rigour of the separate contributions, in the recency of their findings, and in having all technical information at one source. They are up to date yet never out of date, for they offer immediate reference to original work. Medical progress today advances so swiftly that specialist textbooks soon become outdated, and this type of publication is best suited to the needs of the time. This present volume performs the duties of a handbook of psychotropic medication and will find practical use in psychiatry. Research workers in pharmacology and biochemistry will also enjoy access to its technical reports.

\section{CANCER OF THE PHARYNX, LARYNX AND OESOPHAGUS AND ITS SURGICAL TREATMENT}

By Ronald W. Raven, O.B.E.(Mil.), T.D., F.R.C.S. Pp. xiv +292 , fully illustrated. London: Butterworth \& Co. Ltd. 1958. 67s. 6d.

The author has produced a useful contribution to the literature on malignant disease in these situations and it will interest all those who are concerned with its management.

The pharynx, larynx and oesophagus each form separate parts of the book. Each part contains sections on the descriptive anatomy, pathology, clinical features and investigations and the operative surgery, including pre- and post-operative care.

The pharynx - to which nearly two-thirds of the book is devoted-is subdivided into its three anatomical parts and each is dealt with separately in accordance with the above scheme. Apropos of this, it is a pity that the term 'hypopharynx' is used instead of laryngopharynx after employing the terms ' nasopharynx' and 'oropharynx.' 'The section on the descriptive anatomy would have been better if less stereotyped, but the lymphatic drainage has rightly been stressed.

There are some excellent microphotographs in the section on pathology and good radiographs in those dealing with investigations, but some of the photographs of the operative specimens are not well defined.

The descriptions of the operative surgery pertain to the techniques that the author has selected for his own use and form the hard core of the book. They are recorded in a concise and didactic style and are liberally and clearly illustrated. Some of the figures are already familiar, being acknowledged reproductions from other works by the author.

More discussion of alternative operative procedures, albeit minor ones such as neck incisions, would have enhanced these sections.

The management of malignant disease in these situations is often difficult and frequently disappointing, so that many controversial points arise, and the author recognizes that all may not agree with some of his views.

Divergence of opinion is probable in regard to the relative merits of irradiation and surgery, especially in the oropharynx, where the author displays a bias to surgery.

The problems of cervical lymphatic metastases and the plastic repair of pharyngostomes receive special attention, as do the pre-cancerous states of leukoplakia and the Paterson-Kelly syndrome.

The results of the author's surgery are stated and analysed.

Mr. Raven has done good service to place his own experiences on record and to present the many facets of this subject in up-to-date and coherent form.

The artistic dust jacket is significantly macabre in depicting the head of a figure from Rodin's ' Gate of Hell.'

\section{MODERN TRENDS IN DISEASES OF THE VERTEBRAL COLUMN}

Edited by Reginald NASSIM, B.M., F.R.C.P., and H. JACKSON BurRows, M.D., F.R.C.S., F.R.A.C.S. Pp. ix +292 and index, illustrated. London: Butterworth \& Co. Ltd. $1959.75 \mathrm{~s}$.

This is an excellent new book. It consists of a collection of short monographs on various aspects of spinal disease, each one having been written by an acknowledged authority.

The book begins with a short account of the development and anatomy of the vertebral column, 